\title{
IMPROVING THE PERFORMANCE OF WEATHER ROUTING ALGORITHMS
}

\author{
MAXIM V. KOROVKIN \& SERGEI V. POGOZHEV \\ Saint-Petersburg State University, Russia
}

ABSTRACT

This paper presents a framework for solving weather routing problems. Such a problem may be considered as: giving the model of ship dynamics and fuel consumption used for route evaluation, GIS data and weather forecast, which allow to check any route leg for safety, providing the set of routes and satisfying some optimality criteria (e.g. minimal routing time, minimal fuel consumption, specified time of arrival and so on). From the authors' point of view, the searching of such routes may consist of the following steps: 1 . Generation of a set of feasible routes, which are "close" to some extent to desired ones; 2 . Modification of the feasible routes set by applying some transformations to previously found routes; 3 . Fine tuning of route parameters according to search criteria; and 4. Selection of best routes. Several discrete algorithms, proposed for step 1, are compared, so one can chose an appropriate one. Algorithms presented for steps 2 and 3 may be considered as a way of softening the space/time discretization side effects. To compare the efficiency of such algorithms, some common quality measures are presented.

Keywords: weather routing, path finding, optimization.

\section{INTRODUCTION}

For now there are a lot of approaches proposed for different weather routing problems [1], but still there is no common open source benchmark for them such as for various computer vision problems, for example. We consider common weather routing problem in the following exact form based on Sotnikova et al. [2]. Given the spherical rectangle $\Omega_{0}=\left\{(\psi, \lambda) \mid \lambda_{\min } \leq \lambda \leq \lambda_{\max }, \psi_{\min } \leq \psi \leq \psi_{\max }\right\}$, where $\psi$ and $\lambda$ are latitude and longitude respectively, and a number of geographic alarm zones $\Omega_{\mathrm{k}}^{\mathrm{g}}, k=\overline{1 . . n}$, we define static admissible sailing set $\Omega_{s}=\Omega_{0} \backslash \bigcup_{k=1 . . n} \Omega_{\mathrm{k}}^{\mathrm{g}}$. Route must satisfy following condition: $(\psi(t), \lambda(t)) \in \Omega_{s}, t \in\left[t_{0}, t_{1}\right]$ where $t_{0}$ and $t_{1}$ are departure and arrival time respectively. We have to consider dynamic alarm zones $\Omega_{\mathrm{j}}^{d}(t, \psi(t), \lambda(t)), j=\overline{1 . . m}$, which depend not only on weather conditions, but also on vessel motion parameters during the route. Dynamic admissible sailing set is obtained as $\Omega_{d}(t)=\Omega_{0} \backslash U_{j=1 . . m} \Omega_{\mathrm{j}}^{\mathrm{d}}(t)$, and intersection $\Omega_{a}=\Omega_{s} \cap \Omega_{d}$ defines admissible set to search for the best route. Given a departure and arrival points $A\left(\psi_{0}, \lambda_{0}\right) \in \Omega_{a}$ and $B\left(\psi_{1}, \lambda_{1}\right) \in \Omega_{s}$ we define route as a finite sequence of waypoints $A, P_{1}, P_{2}, \ldots, P_{m}, B$, for each of which, except the last one, sailing parameters are set to make the route well defined for corresponding time span. Such sailing parameters may include desired set speed, route leg type (along rhumb line/great circle) and so on. So we obtain some parameter vector $\mu$ together with adjustable waypoint positions $P_{1}, P_{2}, \ldots, P_{m}$ definitely describing selected route $\boldsymbol{r}(t) \leftrightarrow \gamma=\left(P_{1}, \ldots, P_{m}, \mu\right)$. We should note that for different routes length of parameter vector may differ.

Calling this problem formulation "exact" we suppose that for any leg of planned route we can judge whether it is feasible or not and for each problem only one of scalar criteria is used to evaluate the quality of the route built. To select best ones, we formulate:

$$
J(\gamma) \rightarrow \min ,
$$


where vector of parameters $\gamma$ corresponds to feasible route $\boldsymbol{r}(t) \in \Omega_{s} \cap \Omega_{d}(t)$.

This formulation is enough for problems of finding the route with least sailing time/least fuel consumption. For the problem concerning fuel economy with predefined estimated time of arrival $E T A^{*}$ we have to consider additional nonlinear constraint of the form $\operatorname{ETA}(\gamma)=E T A^{*}$.

Proposed approach consists of three stages:

1. feasible path finding;

2. post-processing of feasible routes;

3. optimization.

Since each of these stages may be implemented using different algorithms, providing great variability of routes, one needs to select most suitable combinations of those for given problems.

To evaluate the performance of algorithms the following measures may be introduced in addition to problems' special criteria:

- $\quad$ number of route leg feasibility checks against GIS data, because they are widely used through overall route computation and also one of the most expensive if carried with max accuracy, needed for real world implementations;

- $\quad$ number of vessel speed predictions, given weather data and other sailing conditions along with fixed route splitting time interval used for route statistics calculations.

This choice of efficiency measures may provide independence from GIS/weather forecast information sources and vessel models used, and also from hardware/software setup of testing environment.

\section{PATH FINDING}

According to given discrete route model, two widely used fixed set speed route planning approaches were considered for analysis. Those are modified isochrone [3] and $\mathrm{A}^{*}$. Both of them may be summarized as follows: repeatedly generate some probe legs on top of partly calculated routes, then select some most suitable ones to update partial routes. To reduce memory and computational needs both approaches use some quantization scheme of the search space. There are some notes which may illustrate possible combinatorial nature of algorithm selection problem.

\subsection{Isochrone algorithm notes}

For isochrone algorithm there exists at least two main orthodrome oriented one-dimensional space quantization schemes, according to Hagiwara [3] and Wang et al. [4] (Fig. 1(a), (b)). While the former uses equal angle measure to form bands, the latter uses geographic distance from great circle through departure and destination points. Several initial course selection modes may be considered for newly generated base legs of each band and stage - fixed, previous waypoint [3], departure and destination targeting (Fig. 2). Together with original fixed angle step probe generation scheme variable step scheme [5] and even continuous one [6] may be employed. Probe selection criterion for isochrone may vary from max geodesic distance from departure [3] to generalized ideas of using heuristic function with weighting as of $\mathrm{A}^{*}$ search and iso-cost approach of Tarovik et al. [6]. Fine tuning of algorithm parameters should also include: 
1. selection of time sampling scheme, for example the original idea of James [7] of uniform time steps may be substituted by some non-uniform adaptive one, aiming at reduction of number of route leg checks;

2. selection of course change step and maximum course increment from base leg direction.

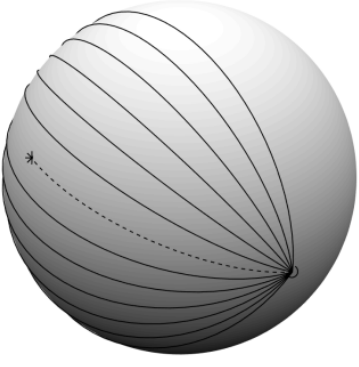

a

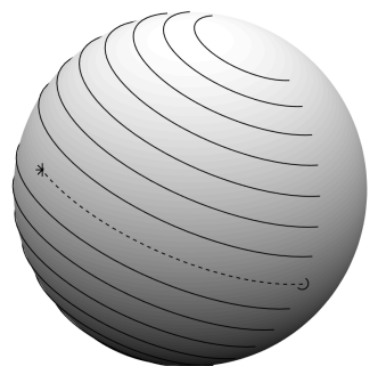

b

Figure 1: Space quantization for isochrone algorithm.

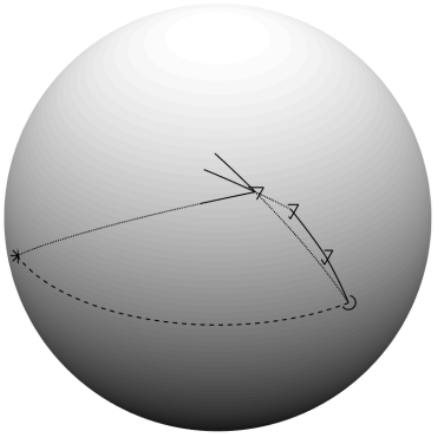

Figure 2: Possible base leg course choices.

Since this algorithm is used only as a preliminary part of route planning, the solution of latter problem may also be performed to overcome the consequences of fixed set speed use. Though the ability of vessel to go back seems inappropriate for motion with the lowest available set speed, for other set speed values the further use of smoothing may provide additional feasible routes. Fig. 3 illustrates the idea of maximum course increment selection for the case of previous waypoint used for legs' base course generation.

\section{$2.2 \mathrm{~A}^{*}$ based algorithms notes}

One of the main questions while using graph based approaches is the choice of corresponding grid system. Since configuration of admissible sailing areas differs greatly for different vessels, online grid construction algorithms were used, that is no nodes and edges of the grid appeared under consideration until needed. Commonly used types of grids may be classified 


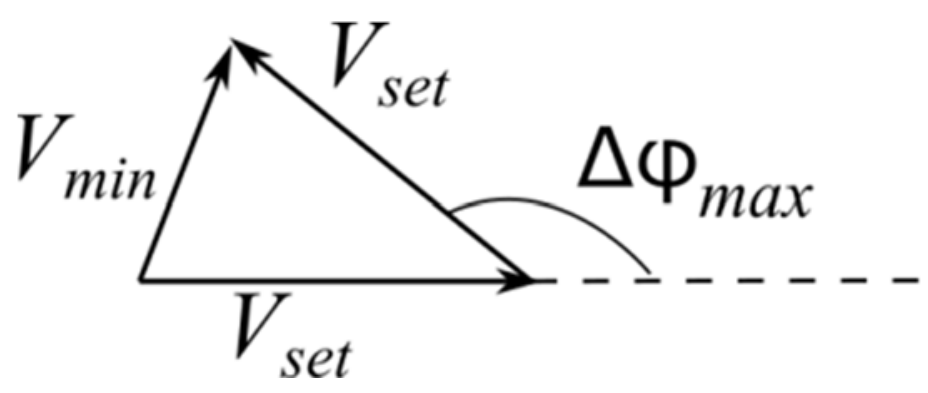

Figure 3: Rule for the selection of max course increment.

as geoid uniform/non-uniform ones, orthodrome oriented/non-orthodrome-oriented. Of those, standard geographic longitude/latitude grid, Mercator projected uniform rectangular grid [8], departure-destination great circle-oriented grids [5], icosahedron-based uniform grid may be mentioned. Analogous to isochrone approach the selection of grid edge size greatly influences the ability of $\mathrm{A}^{*}$ based algorithms to find feasible route. But in contradiction to isochrone approach, graph-based algorithms usually don't "go back" or reuse visited grid nodes.

For each weather routing problem only some range of set speeds is used to produce feasible routes. Given speed step $\Delta V$ least time and least fuel with set ETA problems may use $\left[V_{\text {max }}, V_{\text {max }}-\Delta V, V_{\text {max }}-2 \Delta V, \ldots, V_{\text {min }}\right]$, least fuel problem $\left[V_{\text {cruise }}, V_{\text {cruise }}+\right.$ $\left.\Delta V, V_{\text {cruise }}+2 \Delta V, \ldots, V_{\text {max }}, V_{\text {cruise }}-\Delta V, \ldots, V_{\text {min }}\right]$. To achieve the tradeoff between computation time and quality of planning feasible candidate route quantity threshold may be set to limit number of generated routes. For least fuel with set ETA problem at least two routes should be built, having actual time of arrival just below and above predefined ETA.

\section{SMOOTHING}

The use of space quantizing algorithms for path finding cannot guarantee optimality of found routes in continuous space [9]. There exist two main approaches to overcome this disadvantage. First of them comes from the use of any-angle path finding algorithms [10] instead of above-mentioned ones. The second idea employs smoothing of previously built routes. We may formulate route smoothing problem as: given feasible route R1 characterized by $m$ intermediate waypoints $P_{1}, P_{2}, \ldots, P_{m}$ and parameter vector $\mu_{1}$ produce feasible route R2 with some of original waypoints $P_{i_{1}}, P_{i_{2}}, \ldots, P_{i_{k}}, i_{1}<i_{2}<\cdots<i_{k}$ and parameter vector $\mu_{2}$ having better value of given route evaluation criterion $J$. In the simplest case we try to remove some intermediate points starting from $P_{m}$ taking into account only route length decrease. On each smoothing stage, possibility to remove $P_{k}$ is verified to ensure feasibility of partial route $P_{k-1}, P_{k+1}, \ldots, B$. To do that, check against static constraints of the leg $P_{k-1}, P_{k+1}$ is conducted, and optimization algorithm is employed to select corresponding parameter vector providing the same sailing time on newly formed leg. The latter step may also take into account global optimization target (i.e. we may not let to remove $P_{k}$, since it may increase fuel consumption on the route because of the speed decrease).

Reduced number of route legs may also speed up the following optimization step. Also "oversmoothing" of the route may happen, when reduced number of waypoints doesn't let optimization algorithms to do its work, since the path obtained have not enough "degrees of freedom". Route splitting may be performed in this case to obtain a number of additional intermediate waypoints. 


\section{OPTIMIZATION}

Since smoothed routes consist only of waypoints of original feasible routes, they may be optimized by selection of parameter vector. There exist a number of possible parametrizations of waypoint geographical position to select from: (latitude, longitude) encoding, (distance, course) encoding, two distances along and across departure-destination great circle and so on. Both of them convert the sequence $P_{1}, P_{2}, \ldots, P_{m}$ of intermediate waypoints to $2 \mathrm{~m}$ dimensional bounded vector $\tau$. Having route parameter vector $\mu$ also being bounded, the route may be represented by vector $\gamma=(\tau, \mu), \gamma \in[\underline{\gamma}, \bar{\gamma}]$.

For each route $\boldsymbol{r}(t)$ problem of the form (1) can be converted to the constrained nonlinear optimization problem

$$
\begin{gathered}
J(\gamma) \rightarrow \min , \\
T_{\text {alm }}(\gamma)=0, \\
\gamma \in[\underline{\gamma}, \bar{\gamma}],
\end{gathered}
$$

where $T_{a l m}(\gamma)$ is the time spent in forbidden static and dynamic areas and $J(\gamma)$ is sailing time or fuel consumption. The problem of fuel economy with predefined estimated time of arrival converts to the following:

$$
\begin{gathered}
J_{\text {fuel }}(\gamma) \rightarrow \min , \\
T_{a l m}(\gamma)=0, \\
\operatorname{ETA}(\gamma)=E T A^{*} \\
\gamma \in[\underline{\gamma}, \bar{\gamma}] .
\end{gathered}
$$

To deal with eqns (2) and (3), several algorithms of nonlinear constrained optimization may be used: sequential quadratic programming, penalty and augmented Lagrangian methods [11], genetic algorithms and so on.

\section{EXPERIMENTAL RESULTS}

Several computation experiments were conducted using the model of cargo vessel with the following main parameters:

- $\quad$ length between perpendiculars $-355 \mathrm{~m}$;

- $\quad$ hull breadth $-65 \mathrm{~m}$;

- $\quad$ full draught $-23.0 \mathrm{~m}$;

- $\quad$ waterline coefficient -0.9375 ;

- $\quad$ displacement $-453463.2 \mathrm{t}$;

- $\quad$ max speed - $15 \mathrm{kn}$; cruise speed - $10 \mathrm{kn}$.

First series of experiments concerned an empirical comparison of feasible route-finding algorithms. Seventeen points, most of them being near some ocean ports, were selected, producing 272 possible routes having every combination of these points as a departure and destination. Algorithms used in this test were:

1. isochrone algorithm with equal geodesic distance band width;

2. $\quad \mathrm{A}^{*}$ on equator-oriented latitude-longitude rectangular grid;

3. $A^{*}$ on departure-destination orthodrome oriented latitude-longitude rectangular grid; 
4. $\mathrm{A}^{*}$ on icosahedron based uniform triangular grid;

5. $A^{*}$ on departure-destination orthodrome oriented icosahedron based uniform triangular grid,

and their Theta* counterparts, numbered 6, 7, 8, 9. For isochrone algorithm course angle step was set to $10^{\circ}$, max course change was limited by $112.5^{\circ}$.

Splitting interval was set to 1 hour for route totals calculation.

To be able to compare path finding abilities it is needed to restrict probe step length. Taking into account features of different algorithms, it was done implicitly by setting time base interval $\Delta t$ of three and six hours for each set speed. This value was used directly in isochronal algorithm whereas for latitude-longitude grid angle step was calculated according to the distance $\Delta t \cdot V_{\text {set }}$, sailed by vessel. For icosahedron based uniform triangular grid edge length was selected to be the least one exceeding the distance $\Delta t \cdot V_{\text {set }}$. Table 1 represents route planning results for 3-hour time step along with total number of route leg test calls and speed predictions used to build all 272 routes, including failed routes. It can be seen that the greatest number of routes was found by $\mathrm{A}^{*}$ on rectangular grid, while isochrone algorithm resulted in least number of found routes being most expensive. Compared to $\mathrm{A}^{*}$ algorithm, Theta* has nearly twice number of probe test calls and ten times number of speed predictions for found routes. Using icosahedron based uniform triangular grid results in lesser number of leg feasibility checks due to relatively bigger grid edge length.

Table 1: Feasible path finding success for maximal and cruise set speed, 3-hour time step.

\begin{tabular}{|c|c|c|c|c|c|c|}
\hline \multirow[b]{2}{*}{ Algorithm } & \multicolumn{3}{|c|}{ Maximal speed } & \multicolumn{3}{|c|}{ Cruise speed } \\
\hline & $\begin{array}{l}\text { Number } \\
\text { of } \\
\text { routes } \\
\text { found }\end{array}$ & $\begin{array}{l}\text { Number } \\
\text { of leg } \\
\text { feasibility } \\
\text { checks' } \\
10^{6}\end{array}$ & $\begin{array}{l}\text { Number of } \\
\text { speed } \\
\text { predictions. } \\
10^{7}\end{array}$ & $\begin{array}{c}\text { Number } \\
\text { of } \\
\text { routes } \\
\text { found }\end{array}$ & $\begin{array}{l}\text { Number } \\
\text { of leg } \\
\text { feasibility } \\
\text { checks } \\
10^{6}\end{array}$ & $\begin{array}{c}\text { Number of } \\
\text { speed } \\
\text { predictions } \\
10^{7}\end{array}$ \\
\hline 1 & 114 & 289 & 60.8 & 78 & 343 & 74.9 \\
\hline 2 & 194 & 7.23 & 5.14 & 178 & 13.6 & 5.33 \\
\hline 3 & 200 & 7.88 & 6.21 & 160 & 17.3 & 6.60 \\
\hline 4 & 187 & 3.27 & 2.91 & 153 & 3.28 & 1.87 \\
\hline 5 & 162 & 3.04 & 2.76 & 147 & 2.97 & 1.68 \\
\hline 6 & 170 & 17.3 & 74.7 & 152 & 29.0 & 72.7 \\
\hline 7 & 185 & 17.2 & 82.4 & 150 & 33.8 & 84.1 \\
\hline 8 & 185 & 8.32 & 37.2 & 122 & 7.82 & 15.0 \\
\hline 9 & 158 & 9.59 & 32.2 & 126 & 9.80 & 15.0 \\
\hline
\end{tabular}

Table 2 represents best achievements for mutually found routes (63 on maximal speed and 43 on cruise speed) for 3-hour time step. It can be seen that isochrone algorithm produced highest number of best quality routes. The second place for quality was shared between algorithms of Theta* family, while $\mathrm{A}^{*}$ on triangular grid is least resource consuming.

Efficiency comparison of Theta* and $\mathrm{A}^{*}$ with post smoothing on departure-destination orthodrome oriented latitude-longitude rectangular grid is presented in Table 3. It shows that Theta* algorithm, having online smoothing capabilities, may outperform $A^{*}$ with post smoothing, especially when the cost of leg feasibility check is high. 
Table 2: Mutually found routes quality achievements.

\begin{tabular}{|c|c|c|c|c|c|c|}
\hline & \multicolumn{3}{|c|}{ Maximal speed, number of wins } & \multicolumn{3}{|c|}{ Cruise speed, number of wins } \\
\cline { 2 - 7 } Algorithm & $\begin{array}{c}\text { Least } \\
\text { time }\end{array}$ & $\begin{array}{c}\text { Least } \\
\text { number } \\
\text { of leg } \\
\text { feasibility } \\
\text { checks }\end{array}$ & $\begin{array}{c}\text { Least number } \\
\text { of speed } \\
\text { predictions }\end{array}$ & $\begin{array}{c}\text { Least } \\
\text { fuel } \\
\text { number } \\
\text { of leg } \\
\text { feasibility } \\
\text { checks }\end{array}$ & $\begin{array}{c}\text { Least number } \\
\text { of speed } \\
\text { predictions }\end{array}$ \\
\hline 1 & 48 & 0 & 0 & 19 & 0 & 0 \\
\hline 2 & 0 & 2 & 4 & 0 & 0 & 3 \\
\hline 3 & 0 & 4 & 10 & 0 & 3 & 3 \\
\hline 4 & 0 & 19 & 28 & 0 & 17 & 21 \\
\hline 5 & 1 & 17 & 20 & 0 & 12 & 15 \\
\hline 6 & 4 & 0 & 0 & 11 & 0 & 0 \\
\hline 7 & 1 & 1 & 1 & 9 & 0 & 0 \\
\hline 8 & 7 & 12 & 0 & 3 & 7 & 1 \\
\hline 9 & 1 & 8 & 0 & 1 & 4 & 0 \\
\hline
\end{tabular}

Table 3: A* with smoothing vs. Theta*.

\begin{tabular}{|l|c|c|c|c|c|c|}
\hline \multirow{2}{*}{ Algorithm } & \multicolumn{3}{|c|}{ Least time mode } & \multicolumn{3}{c|}{ Least consumption mode } \\
\cline { 2 - 7 } & $\begin{array}{c}\text { Least } \\
\text { time, } \\
\text { num. of } \\
\text { wins }\end{array}$ & $\begin{array}{c}\text { Number of } \\
\text { leg } \\
\text { feasibility } \\
\text { checks, } \\
\text { wins/totals }\end{array}$ & $\begin{array}{c}\text { Number of } \\
\text { speed } \\
\text { predictions, } \\
\text { wins/totals }\end{array}$ & $\begin{array}{c}\text { Least } \\
\text { fuel, } \\
\text { num. } \\
\text { of } \\
\text { wins }\end{array}$ & $\begin{array}{c}\text { Number of } \\
\text { leg } \\
\text { feasibility } \\
\text { checks, } \\
\text { wins/totals }\end{array}$ & $\begin{array}{c}\text { Number of } \\
\text { speed } \\
\text { predictions, } \\
\text { wins/totals }\end{array}$ \\
\hline $\begin{array}{l}\text { A* post } \\
\text { smoothing }\end{array}$ & 97 & $27 / 3.24 \mathrm{e} 7$ & $116 / 2.40 \mathrm{e} 8$ & 48 & $37 / 5.85 \mathrm{e} 7$ & $106 / 4.69 \mathrm{e} 8$ \\
\hline Theta* & 115 & $185 / 1.58 \mathrm{e} 7$ & $96 / 9.08 \mathrm{e} 8$ & 159 & $170 / 3.60 \mathrm{e} 7$ & $101 / 1.19 \mathrm{e} 9$ \\
\hline
\end{tabular}

In order to use and compare optimization algorithms for weather routing problems, two early stopping modes may be proposed. The first one uses only objective function tolerance as early stopping condition, aiming at best possible quality of the route. The second mode is intended to provide bounded calculation time since the number of objective function evaluations is also fixed.

To take into account nonlinear constraints of eqn (2), the original Nelder-Mead algorithm was modified using the following heuristics. Given initial feasible route, objective function for it is calculated and its value $J\left(\gamma_{0}\right)$ is used to represent the value of $J$ in case of infeasibility of the route represented by parameter vector $\gamma$ :

$$
J(\gamma)= \begin{cases}J\left(\gamma_{0}\right)\left(1+T_{a l m}(\gamma) / T(\gamma)\right), & T_{a l m}(\gamma)>0 \\ J(\gamma), & T_{a l m}(\gamma)=0,\end{cases}
$$

where $T(\gamma)$ is the overall sailing time on the route. Table 4 presents comparison of results of Nelder-Mead bounded algorithm with penalty and sequential quadratic programming used for least time and least consumption problems for feasible routes provided by Theta* algorithm. No limit was set on the number of objective function evaluations. It shows that 
SQP algorithm may provide lesser quality routes having bigger cost variability and overall cost in terms of used efficiency measures.

Table 4: Nelder-Mead bounded vs. SQP optimization algorithms.

\begin{tabular}{|l|c|c|c|c|c|c|}
\hline \multirow{2}{*}{ Algorithm } & \multicolumn{3}{|c|}{ Least time mode } & \multicolumn{3}{c|}{ Least consumption mode } \\
\cline { 2 - 7 } & $\begin{array}{c}\text { Least } \\
\text { time, } \\
\text { num. } \\
\text { of } \\
\text { wins }\end{array}$ & $\begin{array}{c}\text { Number of } \\
\text { leg } \\
\text { feasibility } \\
\text { checks, } \\
\text { wins/totals }\end{array}$ & $\begin{array}{c}\text { Number of } \\
\text { speed } \\
\text { predictions, } \\
\text { wins/totals }\end{array}$ & $\begin{array}{c}\text { Least } \\
\text { fuel, } \\
\text { num. } \\
\text { of } \\
\text { wins }\end{array}$ & $\begin{array}{c}\text { Number of } \\
\text { leg } \\
\text { feasibility } \\
\text { checks, } \\
\text { wins/totals }\end{array}$ & $\begin{array}{c}\text { Number of } \\
\text { speed } \\
\text { predictions, } \\
\text { wins/totals }\end{array}$ \\
\hline $\begin{array}{l}\text { Nelder-Mead } \\
\text { bounded }\end{array}$ & 178 & $54 / 1.97 \mathrm{e} 8$ & $54 / 1.136 \mathrm{e} 9$ & 202 & $36 / 4.41 \mathrm{e} 8$ & $36 / 1.73 \mathrm{e} 9$ \\
\hline SQP & 36 & $160 / 5.66 \mathrm{e} 8$ & $160 / 1.504 \mathrm{e} 9$ & 13 & $173 / 8.87 \mathrm{e} 8$ & $173 / 2.17 \mathrm{e} 9$ \\
\hline
\end{tabular}

\section{ACKNOWLEDGEMENT}

This work is supported by the Russian Foundation for Basic Research under grant 17-0700361 A.

\section{REFERENCES}

[1] Walther, L., Rizvanolli, A., Wendebourg, M. \& Jahn, C., Modeling and optimization algorithms in ship weather routing. International Journal of e-Navigation and Maritime Economy, 4, pp. 31-45, 2016.

[2] Sotnikova, M.V., Veremey, E.I. \& Korovkin, M.V., Transoceanic routes optimization using dynamic properties of ship and weather conditions. Constructive Nonsmooth Analysis and Related Topics (dedicated to the Memory of V.F. Demyanov), St. Petersburg, Russia, 2017

[3] Hagiwara, H., Weather routing of (sail-assisted) motor vessels. $\mathrm{PhD}$ thesis, Technical University of Delft, 1989.

[4] Wang, H., Lia, P., Xueb, Y. \& Korovkin, M.V., Application of improved isochron method in ship's minimum voyage time weather routing. Prikladnaya Matematika. Informatika. Protsessy Upravleniya, 13(3), pp. 286-299, 2017.

[5] Calvert, S., Optimal weather routeing procedures for vessels on trans-oceanic voyages. PhD thesis, University of Plymouth, 1990.

[6] Tarovik, O., Topaj, A., Bakharev, A.A. \& Kondratenko, A., Optimal ice routing of a ship with icebreaker assistance. Applied Ocean Research, 86, pp. 177-187, 2019.

[7] James, R.W., Application of Wave Forecasts to Marine Navigation, U.S. Oceanographic Office: Washington, DC, 1957.

[8] Motte, R., Ship based weather routing (using dynamical meteorology). $\mathrm{PhD}$ thesis, University of Plymouth, 1981.

[9] Daniel, K., Nash, A., Koenig, S. \& Felner, A., Theta*: Any-Angle path planning on grids. Journal of Artificial Intelligence Research, 39, pp. 533-579, 2010.

[10] Uras, T. \& Koenig S., An empirical comparison of any-angle path-planning algorithms. Proceedings of the Eighth International Symposium on Combinatorial Search (SoCS-2015), 2015.

[11] Nocedal, J. \& Wright, S.J., Numerical Optimization, Springer, 2006. 